\title{
Design principles for elementary gene circuits: Elements, methods, and examples
}

\author{
Michael A. Savageau ${ }^{a}$ \\ Department of Microbiology and Immunology, University of Michigan Medical School, \\ 5641 Medical Science Building II, Ann Arbor, Michigan 48109-0620
}

(Received 25 July 2000; accepted for publication 19 December 2000)

The control of gene expression involves complex circuits that exhibit enormous variation in design. For years the most convenient explanation for these variations was historical accident. According to this view, evolution is a haphazard process in which many different designs are generated by chance; there are many ways to accomplish the same thing, and so no further meaning can be attached to such different but equivalent designs. In recent years a more satisfying explanation based on design principles has been found for at least certain aspects of gene circuitry. By design principle we mean a rule that characterizes some biological feature exhibited by a class of systems such that discovery of the rule allows one not only to understand known instances but also to predict new instances within the class. The central importance of gene regulation in modern molecular biology provides strong motivation to search for more of these underlying design principles. The search is in its infancy and there are undoubtedly many design principles that remain to be discovered. The focus of this three-part review will be the class of elementary gene circuits in bacteria. The first part reviews several elements of design that enter into the characterization of elementary gene circuits in prokaryotic organisms. Each of these elements exhibits a variety of realizations whose meaning is generally unclear. The second part reviews mathematical methods used to represent, analyze, and compare alternative designs. Emphasis is placed on particular methods that have been used successfully to identify design principles for elementary gene circuits. The third part reviews four design principles that make specific predictions regarding (1) two alternative modes of gene control, (2) three patterns of coupling gene expression in elementary circuits, (3) two types of switches in inducible gene circuits, and (4) the realizability of alternative gene circuits and their response to phased environmental cues. In each case, the predictions are supported by experimental evidence. These results are important for understanding the function, design, and evolution of elementary gene circuits. (C) 2001 American Institute of Physics. [DOI: 10.1063/1.1349892]

Gene circuits sense their environmental context and orchestrate the expression of a set of genes to produce appropriate patterns of cellular response. The importance of this role has made the experimental study of gene regulation central to nearly all areas of modern molecular biology. The fruits of several decades of intensive investigation have been the discovery of a plethora of both molecular mechanisms and circuitry by which these are interconnected. Despite this impressive progress we are at a loss to understand the integrated behavior of most gene circuits. Our understanding is still fragmentary and descriptive; we know little of the underlying design principles. Several elements of design, each exhibiting a variety of realizations, have been identified among elementary gene circuits in prokaryotic organisms. The use of well-controlled mathematical comparisons has revealed design principles that appear to govern the realization of these elements. These design principles, which make specific predictions supported by experimental data, are important for understanding the normal function of gene circuits; they also are potentially important for developing judicious methods to redirect normal expression for

\footnotetext{
${ }^{a)}$ Electronic mail: savageau@umich.edu
}

biotechnological purposes or to correct pathological expression for therapeutic purposes.

\section{INTRODUCTION}

The gene circuitry of an organism connects its gene set (genome) to its patterns of phenotypic expression. The genotype is determined by the information encoded in the DNA sequence, the phenotype is determined by the contextdependent expression of the genome, and the circuitry interprets the context and orchestrates the patterns of expression. From this perspective it is clear that gene circuitry is at the heart of modern molecular biology. However, the situation is considerably more complex than this simple overview would suggest. Experimental studies of specific gene systems by molecular biologists have revealed an immense variety of molecular mechanisms that are combined into complex gene circuits, and the patterns of gene expression observed in response to environmental and developmental signals are equally diverse.

The enormous variety of mechanisms and circuitry raises questions about the bases for this diversity. Are these variations in design the result of historical accident or have they been selected for specific functional reasons? Are there design principles that can be discovered? By design principle 


\begin{tabular}{|l|l|l|l|l|}
\hline$M_{1} M_{2}$ & $P$ & $G_{1}$ & $G_{2}$ & $T$ \\
\hline
\end{tabular}

FIG. 1. Schematic diagram of a bacterial transcription unit. The structure of the unit consists of two genes $\left(\mathrm{G}_{1}\right.$ and $\left.\mathrm{G}_{2}\right)$, bounded by a promoter sequence $(\mathrm{P})$ and a terminator sequence $(\mathrm{T})$, and preceded by upstream modulator sites $\left(\mathrm{M}_{1}\right.$ and $\left.\mathrm{M}_{2}\right)$ that bind regulators capable of altering transcription initiation. The solid arrow represents the mRNA transcript.

we mean a rule that characterizes some biological feature exhibited by a class of systems such that discovery of the rule allows one not only to understand known instances but also to predict new instances within the class. For many years, most molecular biologists assumed that accident played the dominant role, and the search for rules received little attention. More recently, simple rules have been identified for a few variations in design. Accident and rule both have a role in evolution and their interplay has become clearer in these well-studied cases. This area of investigation is in its infancy and many such questions remain unanswered.

This review article addresses the search for design principles among elementary gene circuits. It reviews first several elements of design for gene circuits, then mathematical methods used to study variations in design, and finally examples of design principles that have been discovered for elementary gene circuits in prokaryotes.

\section{ELEMENTS OF DESIGN AND THE NEED FOR DESIGN PRINCIPLES}

The behavior of an intact biological system can seldom be related directly to its underlying genome. There are several different levels of hierarchical organization that intervene between the genotype and the phenotype. These levels are linked by gene circuits that can be characterized in terms of the following elements of design: transcription unit, input signaling, mode of control, logic unit, expression cascade, and connectivity. Each of these elements exhibits a variety of realizations whose basis is poorly understood.

\section{A. Transcription unit}

A landmark in our understanding of gene circuitry was the discovery by Jacob and Monod of the operon, ${ }^{1}$ the simplest of transcription units. This unit of sequence organization consists of a set of coordinately regulated structural genes (e.g., $G_{1}$ and $G_{2}$ in Fig. 1) that encode proteins, an up-stream promoter site $(\mathrm{P})$ at which transcription of the genes is initiated, and a down-stream terminator site (T) at which transcription ceases. Modulator sites (e.g., $M_{1}$ and $M_{2}$ in Fig. 1) associated with the promoter bind regulatory proteins that influence the rate of transcription initiation (operator sites bind regressors that down-regulate high-level promoters, or initiator sites bind activators that up-regulate lowlevel promoters).

Transcription units are the principal feature around which gene circuits are organized. On the input side, signals in the extracellular (or intracellular) environment are de-
A

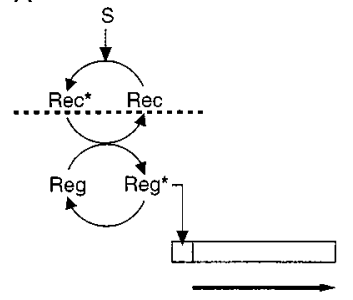

C

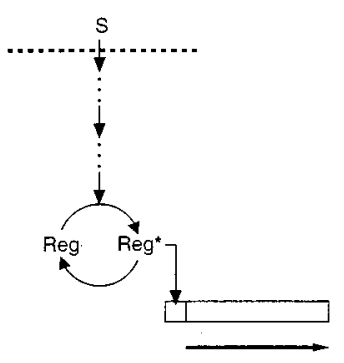

B

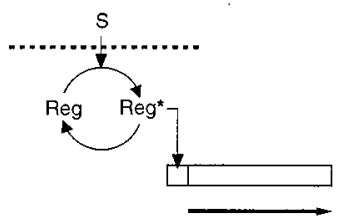

D

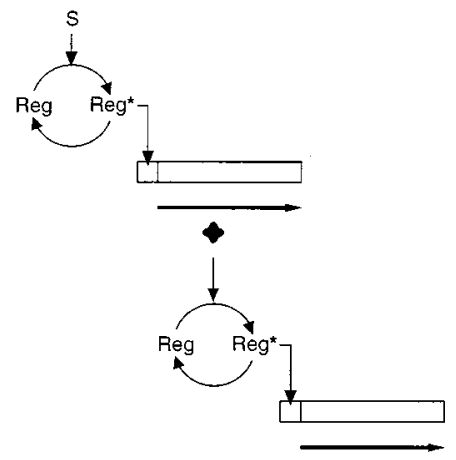

FIG. 2. Input signals for transcription units can arise either from the extracellular environment or from within the cell. $\mathrm{S}$ is a stimulus, Rec and $\mathrm{Rec}^{*}$ are the inactive and active forms of the receptor, and Reg and Reg* are the inactive and active forms of the regulator. (a) Signal transduction from the extracellular environment to an intracellular transcription unit via a twocomponent system. (B) The extracellular signal molecule is transported into the cell where it interacts directly with the regulator of a transcription unit. (c) The signal molecule is transported into the cell where it is transformed via a metabolic pathway to produce a product that interacts with the regulator of a transcription unit. (d) The output signal from one transcription unit is the input signal to another transcription unit within the cell.

tected by binding to specific receptor molecules, which propagate the signal to specific regulatory molecules in a process called transduction, although in many cases the regulator molecules are also the receptor molecules. Regulator molecules in turn bind to the modulator sites of transcription units in one of two alternative modes, and the signals are combined in a logic unit to determine the rate of transcription. On the output side, transcription initiates an expression cascade that yields one or many mRNA products, one or many protein products, and possibly one or many products of enzymatic activity. Thus, the transcription unit emits a fanout of signals, which are then connected in a diverse fashion to the receptors of other transcription units to complete the interlocking gene circuitry.

\section{B. Input signaling}

The input signals for transcription units can arise either from the external environment or from within the cell. When signals originate in the extracellular environment, they often involve binding of signal molecules to specific receptors in the cellular membrane [Fig. 2(a)]. In bacteria, alterations in the membrane-bound receptor are communicated directly to regulator proteins via short signal transduction pathways called "two-component systems." 2 In other cases, signal molecules in the environment are transported across the membrane [Fig. 2(b)], and in some cases are subsequently modified metabolically [Fig. 2(c)], to become signal molecules that bind directly to regulator proteins (in these cases 


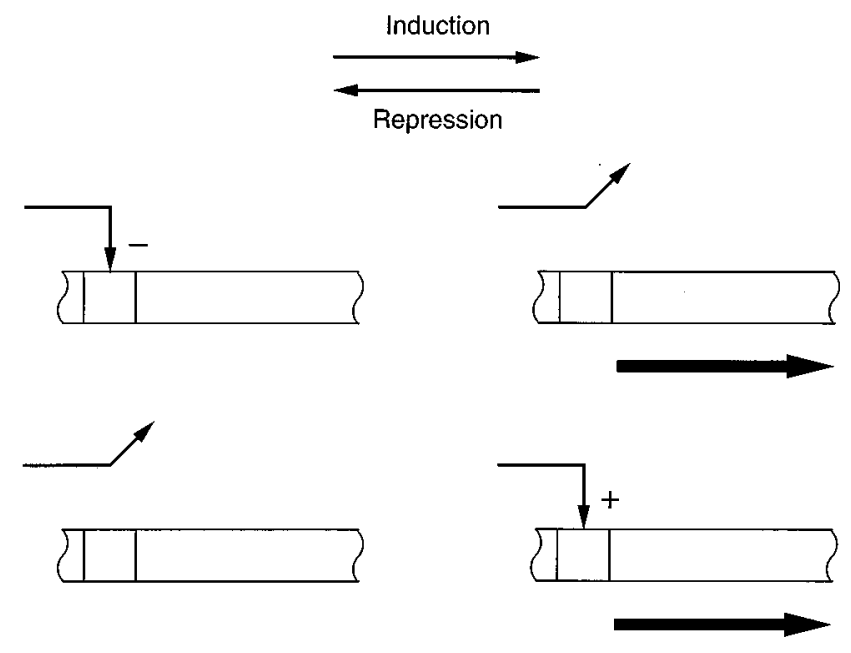

FIG. 3. Alternative modes of gene control. The top panels illustrate the negative mode of control in which the bias for expression is $\mathrm{ON}$ in the absence of the regulator, and regulation is achieved by modulating the effectiveness of a negative element. The bottom panels illustrate the positive mode of control in which the bias for expression is OFF in the absence of the regulator, and regulation is achieved by modulating the effectiveness of a positive element. The solid arrow represents the mRNA transcript. In each case, induction by the addition of a specific inducer causes the state of the system to shift from the left to the right, whereas repression by the addition of a specific co-repressor causes the state of the system to shift from right to left.

the receptor and regulator are one and the same molecule). When signals arise from other transcription units within the cell, the regulator can be the direct output signal from such a transcription unit [Fig. 2(d)]. It can also be the terminus of a signal transduction pathway in which the upstream signal is the output from such a transcription unit. Thus, the input signals for transcription units are ultimately the regulators, whether signals are received from the extracellular or intracellular environment. The regulators in most cases are protein molecules, although this function can be preformed in some cases by other types of molecules such as anti-sense RNA.

\section{Mode of control}

Regulators exert their control over gene expression by acting in one of two different modes (Fig. 3). ${ }^{3}$ In the positive mode, they stimulate expression of an otherwise quiescent gene, and induction of gene expression is achieved by supplying the functional form of the regulator. In the negative mode, regulators block expression of an otherwise active gene, and induction of gene expression is achieved by removing the functional form of the regulator. Each of these two designs (positive or negative) requires the transcription unit to have the appropriate modulator site (initiator type or operator type) and promoter function (low level or high level).

Variations in the level of the functional form of the regulator can be achieved in different ways. Regulator molecules can have a constant or constitutive level of expression. In this case, the functional form of the regulator is created or destroyed by molecular alterations associated with the binding of specific ligands (inducers or co-regressors). In other

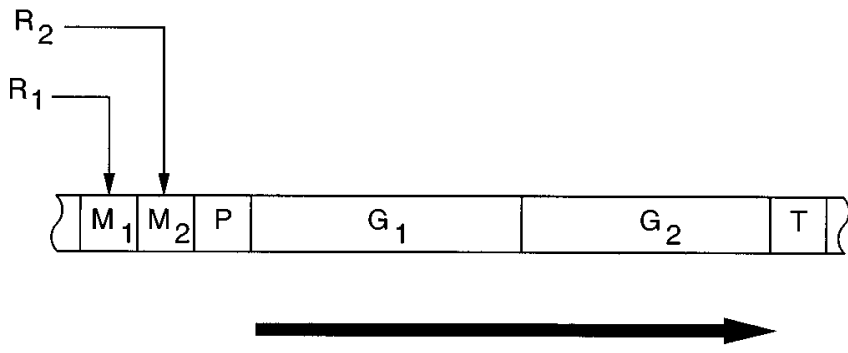

\begin{tabular}{llllll}
\hline \multicolumn{2}{c}{ Mode } & \multicolumn{2}{c}{ Presence } & \multicolumn{2}{c}{ Expression } \\
\hline$R_{1}$ & $R_{2}$ & $R_{1}$ & $R_{2}$ & AND & OR \\
\hline+ & - & Yes & Yes & OFF & ON \\
& & Yes & No & ON & ON \\
& No & Yes & OFF & OFF \\
& No & No & OFF & ON \\
& & & & & \\
\end{tabular}

FIG. 4. Logic unit with two inputs. The transcription unit is described in Fig. 1, the regulator $R_{1}$ interacts with the modulator site $M_{1}$ via the positive mode, the regulator $R_{2}$ interacts with the modulator sites $M_{2}$ via the negative mode, and the signals are combined by a simple logical function. The logic table is provided for the logical AND and logical OR functions.

cases, the regulator is always in the functional form, and its level of expression varies as the result of changes in its rate of synthesis or degradation. These different ways of realizing variations in the functional form of the regulator are found for both positive and negative modes of control.

\section{Logic unit}

The control regions associated with transcription units may be considered the logic unit where input signals from various regulators are integrated to govern the rate of transcription initiation. There are two lines of evidence suggesting that most transcription units in bacteria have only a few regulatory inputs. First, the early computational studies of Stuart Kauffman using abstract random Boolean networks suggested that two or three inputs per transcription unit were optimal. ${ }^{4}$ If the number of inputs was fewer on average, the behavior of the network was too fixed; whereas if the number was greater on average, the behavior was too chaotic. The optimal behavior associated with a few inputs often is described as "operating at the edge of chaos.", Second, with the arrival of the genomic era and the sequencing of the complete genome for a number of bacteria, there is now experimental evidence regarding the distribution of inputs per transcription unit. The sequence for Escherichia coli ${ }^{6}$ has shown that the number of modulator sites located near the promoters of transcription units is on average approximately two to three. ${ }^{7}$ The large majority have two and a few have as many as five.

A simple logic unit is illustrated in Fig. 4 for the case with two inputs. This example includes the classical lactose 
(lac) operon of E. coli, which has a positive and a negative regulator; the AND function is the logical operator by which these signals are combined. ${ }^{8}$ The logic units of eukaryotes can be considerably more complex. ${ }^{9}$

\section{E. Expression cascades}

Expression cascades produce the output signals from transcription units. They typically reflect the flow of information from DNA to RNA to protein to metabolites, which has been called the "Central Dogma" of molecular biology. The initial output of a transcription unit is an mRNA molecule that has a sequence complementary to the transcribed DNA strand. The mRNA in turn is translated to produce the encoded protein product. The protein product in many instances is an enzyme, which in turn catalyzes a specific reaction to produce a particular metabolic product. This in skeletal form is the expression cascade that is initiated by signals affecting a transcription unit (Fig. 5).

There are many variations on this theme. There can be additional stages in such cascades and each of the stages is a potential target for regulation. For example, the cascade might include posttranscriptional or posttranslational stages in which products are processed before the next stage in the cascade. The cascade can also include a stage in which a RNA template is used to transcribe a complementary DNA copy, as is the case with retroviruses and retrotransposons.

There can be multiple products produced at each stage of such cascades. For example, several different mRNA molecules can arise from the same transcription unit by regulation of transcription termination. Several different proteins can be synthesized from the same mRNA and this is often the case in bacteria. Several metabolic products can be produced by a given multifunctional enzyme, depending upon its modular composition. Thus, transcription units can be considered to emit a fan of output signals.

\section{F. Connectivity}

The connectivity of gene circuits, defined as the manner in which the outputs of transcription units are connected to the inputs of other transcription units, varies enormously. The evidence for $E$. coli suggests a fairly narrow distribution of input connections with a mean of two to three, whereas the distribution of output connections has a wider distribution with some transcription units having as many as $\mathbf{5 0}$ output connections. A large number of the connections involve

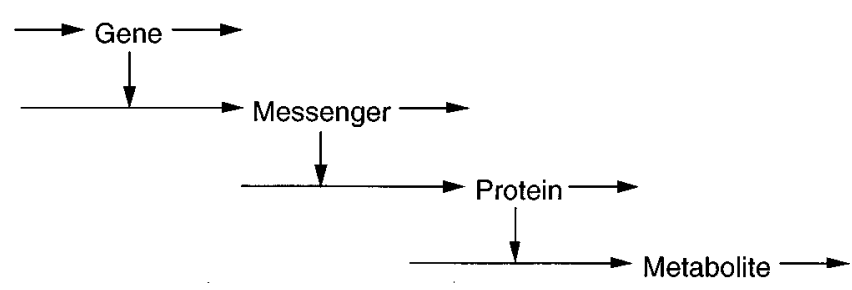

FIG. 5. Expression cascade that propagates signals in three stages from DNA to mRNA to enzymes to small molecular weight signaling molecules. Additional stages are possible, and each stage can give rise to multiple output signals.

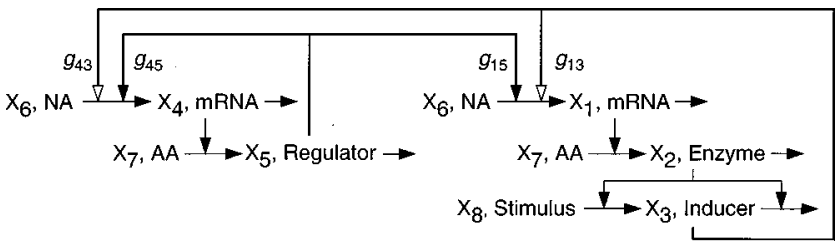

FIG. 6. Connectivity by which expression cascades become coupled. Elementary circuit consisting of a regulator cascade on the left and an effector cascade on the right. The protein product that is the output of the left cascade is a regulator of both transcription units, and the metabolic intermediate that is an output of the right cascade is an inducer that modulates the effectiveness of the regulator at each transcription unit.

regulator proteins modulating expression of the transcription unit in which they are encoded, a form of regulation termed autogenous. ${ }^{10}$ Another common form of connection involves the coupling of expression cascades for an effector function and for its associated regulator. ${ }^{11}$ Such couplings are called elementary gene circuits and an example is represented schematically in Fig. 6.

Connectivity provides a way of coordinating the expression of related functions in the cell. ${ }^{12}$ The operon, a transcription unit consisting of several structural genes that are transcribed as a single polycistronic mRNA, provides one way of coordinating the expression of several genes. Another way is to have each gene in a separate transcription unit and have all the transcription units connected to the same regulatory input signal. Such a set of coordinately regulated transcription units is known as a regulon. Other, and more flexible, ways also exist. For example, when signals from several regulators are assembled in a combinatorial fashion to govern a collection of transcription units, each with its own logic unit, diverse patterns of gene expression can be orchestrated in response to a variety of environmental contexts.

\section{METHODS FOR COMPARING DESIGNS TO REVEAL DESIGN PRINCIPLES}

Several different approaches have been used to analyze and compare gene circuits, and each has contributed in different ways to our understanding. Here I need only mention three of the approaches that have been dealt with in greater detail elsewhere.

\section{A. Types of models}

Simplified models based on random Boolean networks have been used to explore properties that are likely to be present with high probability regardless of mechanistic details or evolutionary history. These tend to be discrete/ deterministic models that permit efficient computational exploration of large populations of networks, which then permit statistical conclusions to be drawn. The work of Kauffman provides an example of this approach. ${ }^{4}$ The elements of design emphasized in this approach are the input logic units and the connectivity, and properties of the network are examined as a function of network size.

Detailed mechanistic models have been used to test our understanding of particular gene circuits. The goal is to represent the detailed behavior as faithfully as possible. A mixture of discrete/continuous/deterministic/stochastic model el- 
ements might be used, depending upon the particular circuit. These are computationally intensive and require numerical values for the parameters, and detailed quantitative comparisons with experimental data are important means of validation. The work of Arkin and colleagues illustrates this approach. ${ }^{13}$ The elements of design emphasized in this approach are all those that manifest themselves in the particular circuit being modeled.

Generic models for specific classes of circuits have been used to identify design principles for each class. The aim of these models is to capture qualitative features of behavior that hold regardless of the specific values for the parameters and hence that are applicable to the entire class being characterized. These tend to be continuous/deterministic models with a regular formal structure that facilitates analytical and numerical comparisons. Examples of this approach will be reviewed below in Sec. IV. The elements of design that tend to be emphasized in this approach are expression cascades, modes of control, input logic units, and connectivity.

\section{B. A comparative approach to the study of design}

The elucidation of design principles for a class of circuits requires a formalism to represent alternative designs, methods of analysis capable of predicting behavior, and methods for making well-controlled comparisons.

\section{Canonical nonlinear representation}

The power-law formalism combines nonlinear elements having a very specific structure (products of power laws) with a linear operator (differentiation) to form a set of ordinary differential equations, which are capable of representing any suitably differentiable nonlinear function. This makes it an appropriate formalism for representing alternative designs.

The elements of the power-law formalism are nonlinear functions consisting of simple products of power-law functions of the state variables ${ }^{14}$

$$
v_{i}(\mathbf{X})=\alpha_{i} X_{1}^{g_{i 1}} X_{2}^{g_{i 2}} X_{3}^{g_{i 3} \cdots X_{n}}{ }^{g_{i n}} .
$$

The two types of parameters in this formalism are referred to as multiplicative parameters $\left(\alpha_{i}\right)$ and exponential parameters $\left(g_{i j}\right)$. They also are referred to as rate-constant parameters and kinetic-order parameters, since these are accepted terms in the context of chemical and biochemical kinetics. The multiplicative parameters are non-negative real, the exponential parameters are real, and the state variables are positive real.

Although the nonlinear behavior exhibited by these nonlinear elements is fairly impressive, it does not represent the full spectrum of nonlinear behavior that is characteristic of the power-law formalism. When these nonlinear elements are combined with the differential operator to form a set of ordinary differential equations they are capable of representing any suitably differentiable nonlinear function. The two most common representations within the power-law formalism are generalized-mass-action (GMA) systems $d X_{i} / d t=\sum_{k=1}^{r} \alpha_{i k} \prod_{j=1}^{n+m} X_{j}^{g_{i j k}}-\sum_{k=1}^{r} \beta_{i k} \prod_{j=1}^{n+m} X_{j}^{h_{i j k}}, \quad i=1, \ldots, n$,

and synergistic $(\mathrm{S})$ systems

$$
d X_{i} / d t=\alpha_{i} \prod_{j=1}^{n+m} X_{j}^{g_{i j}}-\beta_{i} \prod_{j=1}^{n+m} X_{j}^{h_{i j}}, \quad i=1, \ldots, n .
$$

The derivatives of the state variables with respect to time $t$ are given by $d X_{i} / d t$. The $\alpha$ and $g$ parameters are defined as in Eq. (1) and are used to characterize the positive terms in Eqs. (2) and (3), whereas the $\beta$ and $h$ parameters are similarly defined and are used to characterize the negative terms. There are in general $n$ dependent variables, $m$ independent variables, and a maximum of $r$ terms of a given sign. The resulting power-law formalism can be considered a canonical nonlinear representation from at least three different perspectives: fundamental, recast, and local. ${ }^{15}$

As the natural representation of the elements postulated to be fundamental in a variety of fields, the power-law formalism can be considered a canonical nonlinear representation. There are a number of representations that are considered fundamental descriptions of the basic entities in various fields. Four such representations that are extensively used in chemistry, population biology, and physiology are massaction, Volterra-Lotka, Michaelis-Menten, and linear representations. These are, in fact, special cases of the GMAsystem representation, ${ }^{15}$ which, as noted earlier, is one of the two most common representations within the general framework of the power-law formalism. Although, the power-law formalism can be considered a fundamental representation of chemical kinetic events, this is not the most useful level of representation for comparing gene circuits because it is much too detailed and values for many of the elementary parameters will not be available. Nor does the structure of the GMA equations lend itself to general symbolic analysis.

As a recast description, the power-law formalism can be considered a canonical nonlinear representation in nearly every case of physical interest. This is because any nonlinear function or set of differential equations that is a composite of elementary functions can be transformed exactly into the power-law formalism through a procedure called recasting. ${ }^{16}$ This is a well-defined procedure for generating a globally accurate representation that is functionally equivalent to the original representation. In this procedure one trades fewer equations with more complex and varied forms of nonlinearity for more equations with simpler and more regular nonlinear forms. Although the power-law formalism in the context of recasting has important uses and allows for efficient numerical solution of differential equations, this again is not the most useful level of representation for comparing alternative designs for gene circuits because it does not lend itself to general systematic analysis.

As a local description, the power-law formalism can be considered a canonical nonlinear representation that is typically accurate over a wider range of variation than the corresponding linear representation. The state variables of a system can nearly always be defined as positive quantities. Therefore, functions of the state variables can be represented 
equivalently in a logarithmic space-i.e., a space in which the logarithm of the function is a function of the logarithms of the state variables. This means that a Taylor series in logarithmic space can also be used as a canonical representation of the function. If the variables make only small excursions about their nominal operating values, then this series can be truncated at the linear terms, transformed back into Cartesian coordinates, and expressed in the power-law formalism. Thus, Taylor's theorem gives a rigorous justification for the local power-law formalism and specific error bounds within which it will provide an accurate representation.

A rigorous and systematic analysis of the second-order contributions to the local power-law representation has been developed by Salvador. ${ }^{17,18}$ This analysis provides a valuable approach for making rational choices concerning model reduction. By determining the second-order terms in the power-law approximation of a more complex model one can determine those parts of the model that are accurately represented by the first-order terms. These parts of the model can be safely represented by the local representation; those parts that would not be represented with sufficient accuracy can then be dealt with in a variety of ways, including a more fundamental model or a recast model, either of which would leave the resulting model within the power-law representation.

The local S-system representation within the power-law formalism has proved to be more fruitful than the local GMA-system representation because of its accuracy and structure. It is typically more accurate because it allows for cancellation of systematic errors. ${ }^{19,20}$ It has a more desirable structure from the standpoint of general symbolic analysis: there is an analytical condition for the existence of a steady state, an analytical solution for the steady state, and an analytical condition that is necessary for the local stability of the steady state. The regular structure and tractability of the S-system representation is an advantage in systematic approaches for inferring the structure of gene networks from global expression data. ${ }^{21}$

The S-system representation, like the linear and Volterra-Lotka representations, exhibits the same structure at different hierarchical levels of organization. ${ }^{22} \mathrm{We}$ call this the telescopic property of the formalism. Only a few formalisms are known to exhibit this property. A canonical formalism that provides a consistent representation across various levels of hierarchical organization in space and time has a number of advantages. For example, consider a system described by a set of S-system equations with $n$ dependent variables. Now suppose that the variables of the system form a temporal hierarchy such that $k$ of them determine the temporal behavior of the system. The $n-k$ "fast" variables are further assumed to approach a quasi-steady state in which they are now related to the $k$ temporally dominant variables by power-law equations. When these relationships are substituted into the differential equations for the temporally dominant variables, a new set of differential equations with $k$ dependent variables is the result. This reduced set is also an $\mathrm{S}$-system; that is, the temporally dominant subsystem is represented within the same power-law formalism. Thus, the same methods of analysis can be applied at each hierarchical level.

Power-law expressions are found at all hierarchical levels of organization from the molecular level of elementary chemical reactions to the organismal level of growth and allometric morphogenesis. ${ }^{15}$ This recurrence of the power law at different levels of organization is reminiscent of fractal phenomena, which exhibit the same behavior regardless of scale. ${ }^{23}$ In the case of fractal phenomena, it has been shown that this self-similar property is intimately associated with the power-law expression. ${ }^{24}$ Hence, it is not surprising that the power-law formalism should provide a canonical representation with telescopic properties appropriate for the characterization of complex nonlinear systems.

Finally, piecewise power-law representations provide a logical extension of the local power-law representation. The piecewise linear representation has long been used in the temporal analysis of electronic circuits. ${ }^{25}$ It simplifies the analysis, converting an intractable nonlinear system of equations into a series of simple linear systems of equations whose behavior, when pieced together, is capable of closely approximating that of the original system. A different use of an analogous piecewise representation was developed by Bode to simplify the interpretation of complex rational functions that characterize the frequency response of electronic circuits. ${ }^{26}$ This type of Bode analysis was adapted for interpretation of the rational functions traditionally used to represent biochemical rate laws ${ }^{27}$ and then developed more fully into a systematic power-law formalism for the local representation of biochemical systems consisting of many enzymatic reactions. ${ }^{15}$ In analogy with traditional piecewise linear analysis, a piecewise power-law representation has been developed and used to analyze models of gene circuitry (see Sec. IV C). This form of representation greatly simplifies the analysis; it also captures the essential nonlinear behavior more directly and with fewer segments than would a piecewise linear representation.

\section{Methods of analysis}

The regular, systematic structure of the power-law formalism implies that methods developed to solve efficiently equations having this form will be applicable to a wide class of phenomena. This provides a powerful stimulus to search for such methods. The potential of the power-law formalism in this regard has yet to be fully exploited. The following are some examples of generic methods that have been developed for analysis within the framework of the power-law formalism.

The simplicity of the local S-system representation has led to the most extensive development of theory, methodology, and applications within the power-law formalism. ${ }^{28} \mathrm{In}$ deed, as discussed in Sec. III B 1, the local S-system representation allows the derivation of important systemic properties that would be difficult, if not impossible, to deduce by other means. These advances have occurred because it was recognized from the beginning that the steady-state analysis of S-systems reduces to conventional linear analysis in a logarithmic space. Hence, one was able to exploit the powerful methods already developed for linear systems. For 
example, S-systems have an explicit analytical solution for the steady state. ${ }^{14,27}$ The condition for the existence of such a steady state reduces to the evaluation of a simple determinant involving the exponential parameters of the S-system. Local stability is determined by two critical conditions, one involving only the exponential parameters and the other involving these as well as the multiplicative parameters. Steady-state (logarithmic) gain matrices provide a complete network analysis of the signals that propagate through the system. Similarly, steady-state sensitivity matrices provide a complete sensitivity analysis of the parameters that define the system and its robustness. The linear structure also permits the use of well-developed optimization theory such as the simplex method. ${ }^{29}$

Analytical solutions for the local dynamic behavior are available, including eigenvalue analysis for characterization of the relaxation times. ${ }^{30}$ The regular structure also allows the conditions for Hopf bifurcation to be expressed as a simple formula involving the exponential parameters. ${ }^{31}$ However, S-systems are ultimately nonlinear systems and so there is no analytical solution for dynamic behavior outside the range of accurate linear representation, which is more restrictive than the range of accurate power-law representation. Determination of the local dynamic behavior within this larger range, and the determination of global dynamic behavior, requires numerical methods.

An example of what can be done along these lines is the efficient algorithm developed for solving differential equations represented in the canonical power-law formalism. ${ }^{32}$ This algorithm, when combined with recasting, ${ }^{16}$ can be used to obtain solutions for rather arbitrary nonlinear differential equations. More significantly, this canonical approach has been shown to yield solutions in shorter time and with greater accuracy, reliability, and predictability than is typically possible with other methods. This algorithm can be applied to other canonical formalisms as well as to all representations within the power-law formalism. This algorithm has been implemented in a user-friendly program call PLAS (Power-Law Analysis and Simulation), which is available on the web (http://correio.cc.fc.ul.pt/ aenf/plas.html).

Another example is an algorithm based on the S-system representation that finds multiple roots of nonlinear algebraic equations. ${ }^{33,34}$ Recasting allows one to express rather general nonlinear equations in the GMA-system representation within power-law formalism. The steady states of the GMAsystem, which correspond to the roots of the original algebraic equation, cannot be obtained analytically. However, these power-law equations can be solved iteratively using a local S-system representation, which amounts to a Newton method in logarithmic space. Each step makes use of the analytical solution that is available with the S-system representation (see earlier in this work). The method is robust and converges rapidly. ${ }^{33}$ Choosing initial conditions to be the solution for an S-system with terms selected in a combinatorial manner from among the terms of the larger GMAsystem has been shown to find many, and in some cases all, of the roots for the original equations. ${ }^{34}$

\section{Mathematically controlled comparison of alternatives}

The existence of an explicit solution allows for the analytical specification of systemic constraints or invariants that provide the basis for the method of mathematically controlled comparisons. ${ }^{10,11,27,30,35,36}$ The method involves the following steps. (1) The alternatives being compared are restricted to having differences in a single specific process that remains embedded within its natural milieu. (2) The values of the parameters that characterize the unaltered processes of one system are assumed to be strictly identical with those of the corresponding parameters of the alternative system. This equivalence of parameter values within the systems is called internal equivalence. It provides a means of nullifying or diminishing the influence of the background, which in complex systems is largely unknown. (3) Parameters associated with the changed process are initially free to assume any value. This allows the creation of new degrees of freedom. (4) The extra degrees of freedom are then systematically reduced by imposing constraints on the external behavior of the systems, e.g., by insisting that signals transmitted from input (independent variables) to output (dependent variables) be amplified by the same factor in the alternative systems. In this way the two systems are made as nearly equivalent as possible in their interactions with the outside environment. This is called external equivalence. (5) The constraints imposed by external equivalence fix the values of the altered parameters in such a way that arbitrary differences in systemic behavior are eliminated. Functional differences that remain between alternative systems with maximum internal and external equivalence constitute irreducible differences. (6) When all degrees of freedom have been eliminated, and the alternatives are as close to equivalent as they can be, then comparisons are made by rigorous mathematical and computer analyses of the alternatives.

Two key features of this method should be noted. First, because much of the analysis can be carried out symbolically, the results are often independent of the numerical values for particular parameters. This is a marked advantage because one does not know, and in many cases it would be impractical to obtain, all the parameter values of a complex system. Second, the method allows one to determine the relative optima of alternative designs without actually having to carry out an optimization (i.e., without having to determine explicit values for the parameters that optimize the performance of a given design). If one can show that a given design with an arbitrary set of parameter values is always superior to the alternative design that has been made internally and externally equivalent, whether or not the set of parameter values represents an optimum for either design, then one has proved that the given design will be superior to the alternative even if the alternative were assigned a parameter set that optimized its performance. This feature is a decided advantage because one can avoid the difficult procedure of optimizing complex nonlinear systems.

The method of mathematically controlled comparison has been used for some time to determine which of two alternative regulatory designs is better according to specific quantitative criteria for functional effectiveness. In some 
cases, as noted above, the results obtained using this technique are general and qualitatively clear cut, i.e., one design is always better than another, independent of parameter values. For example, consider some systemic property, say a particular parameter sensitivity, whose magnitude should be as small as possible. In many cases, the ratio of this property in the alternative design relative to that in the reference design has the form $R=A /(A+B)$ where $A$ and $B$ are positive quantities with a distinct composition involving many individual parameters. Such a ratio is always less than one, which indicates that the alternative design is superior to the reference design with regard to this desirable property. In other cases, the results might be general but difficult to demonstrate because the ratio has a more complex form, and comparisons made with specific values for the parameters can help to clarify the situation. In either of these cases, comparisons made with specific values for the parameters also can provide a quantitative answer to the question of how much better one of the alternatives might be.

In contrast to the cases discussed previously, in which a clear-cut qualitative difference exists, a more ambiguous result is obtained when either of the alternatives can be better, depending on the specific values of the parameters. For example, the ratio of some desirable systemic property in the alternative design relative to that in the reference design has the form $R=(A+C) /(A+B)$, where $A, B$, and $C$ are positive quantities with a distinct composition involving many individual parameters. For some values of the individual parameters $C>B$ and for other values $C<B$, so there is no clear-cut qualitative result. A numerical approach to this problem has recently been developed that combines the method of mathematically controlled comparison with statistical techniques to yield numerical results that are general in a statistical sense. ${ }^{37}$ This approach retains some of the generality that makes mathematically controlled comparison so attractive, and at the same time provides quantitative results that are lacking in the qualitative approach.

\section{EXAMPLES OF DESIGN PRINCIPLES FOR ELEMENTARY GENE CIRCUITS}

Each design feature of gene circuits allows for several differences in design. Our goal is to discover the design principles, if such exist, that would allow one to make predictions concerning which of the different designs would be selected under various conditions. For most features, the design principles are unknown, and we are currently unable to predict which design among a variety of well-characterized designs might be selected in a given context. In a few cases, as reviewed later, principles have been uncovered. There are simple rules that predict whether the mode of control will be positive or negative, whether elementary circuits will be directly coupled, inversely coupled, or uncoupled, and whether gene expression will switch in a static or dynamic fashion. More subtle conditions relate the logic of gene expression to the context provided by the life cycle of the organism.

\section{A. Molecular mode of control}

A simple demand theory based on selection allows one to predict the molecular mode of gene control. This theory
TABLE I. Predicted correlation between molecular mode of control and the demand for gene expression in the natural environment.

\begin{tabular}{cll}
\hline \hline \multirow{2}{*}{ Demand for expression } & \multicolumn{2}{c}{ Mode of control } \\
\cline { 2 - 3 } & Positive & Negative \\
\hline High & Selected & Lost \\
Low & Lost & Selected \\
\hline \hline
\end{tabular}

states that the mode of control is correlated with the demand for gene expression in the organism's natural environment: positive when demand is high and negative when demand is low. Development of this theory involved elucidating functional differences, determining the consequences of mutational entropy (the tendency for random mutations to degrade highly ordered structures rather than contribute to their formation), and examining selection in alternative environments.

Detailed analysis involving mathematically controlled comparisons demonstrates that model gene circuits with the alternative modes of control behave identically in most respects. However, they respond in diametrically opposed ways to mutations in the control elements themselves. ${ }^{27} \mathrm{Mu}-$ tational entropy leads to loss of control in each case. However, this is manifested as super-repressed expression in circuits with the positive mode of control, and constitutive expression in circuits with the negative mode. The dynamics of mixed populations of organisms that harbor either the mutant or the wild-type control mechanism depend on whether the demand for gene expression in the environment is high or low. ${ }^{38}$ The results are summarized in Table I. The basis for these results can be understood in terms of the following qualitative argument involving extreme environments.

A gene with a positive mode of control and a high demand for its expression will be induced normally if the control mechanism is wild type. It will be uninduced if the control mechanism is mutant, and, since expression cannot meet the demand in this case, the organism harboring the mutant mechanism will be selected against. In other words, the functional positive mode of control will be selected when mutant and wild-type organisms grow in a mixed population. On the other hand, in an environment with a low demand for expression, the gene will be uninduced in both wild-type and mutant organisms and there will be no selection. Instead, the mutants will accumulate with time because of mutational entropy, and the wild-type organisms with the functional positive mode of control will be lost.

The results for the negative mode of control are just the reverse. A gene with a negative mode of control and a low demand for its expression will be uninduced normally if the control mechanism is wild type. It will be constitutively induced if the control mechanism is mutant, and, since inappropriate expression in time or space tends to be dysfunctional, the organism harboring the mutant mechanism will be selected against. In other words, the functional negative mode of control will be selected when mutant and wild-type organisms grow in a mixed population. On the other hand, in an environment with a high demand for expression, the gene will be induced in both wild-type and mutant organisms and 
TABLE II. General predictions regarding the mode of control for regulation of cell-specific functions in differentiated cell types. ${ }^{\text {a }}$

\begin{tabular}{cll}
\hline \hline & \multicolumn{2}{c}{ Cell-specific functions } \\
\cline { 2 - 3 } Cell type & \multicolumn{1}{c}{$\mathrm{A}$} & \multicolumn{1}{c}{$\mathrm{B}$} \\
\hline $\mathrm{A}$ & Positive & Negative \\
$\mathrm{B}$ & Negative & Positive \\
\hline \hline
\end{tabular}

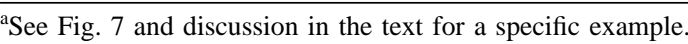

there will be no selection. Instead, the mutants will accumulate with time because of mutational entropy, and the wildtype organisms with the functional negative mode of control will be lost.

The predictions of demand theory are in agreement with nearly all individual examples for which both the mode of control and the demand for expression are well-documented. ${ }^{39}$ On the basis of this strong correlation, one can make predictions concerning the mode of control when the natural demand for expression is known, or vice versa. Moreover, when knowledge of cellular physiology dictates that pairs of regulated genes should be subject to the same demand regime, even if it is unknown whether the demand in the natural environment is high or low, then demand theory allows one to predict that the mode of control will be of the same type for both genes. Conversely, when such genes should be subject to opposite demand regimes, and again even if it is unknown whether the demand in the natural environment is high or low, then demand theory allows one to predict that the mode of control will be of the opposite type for these genes. The value of such predictions is that once the mode of control is determined experimentally for one of the two genes, one can immediately predict the mode of control for the other.

Straightforward application of demand theory to the control of cell-specific functions in differentiated cell types not only makes predictions about the mode of control for these functions in each of the cell types, but also makes the surprising prediction that the mode of control itself ought to undergo switching during differentiation from one cell type to another. ${ }^{40}$ Table II summarizes the general predictions, and Fig. 7 provides a specific example of a simple model system, cells of Escherichia coli infected with the temperate bacteriophage $\lambda$, that fulfills these predictions. During lytic growth (cell type A in Table II), the lytic functions (Aspecific functions) are in high demand and are predicted to involve the positive mode of control. Indeed, they are controlled by the $\mathrm{N}$ gene product, which is an anti-terminator exercising a positive mode of control. At the same time, the lysogenic functions (B-specific functions) are in low demand and are predicted to involve the negative mode of control. In this case, they are controlled by the CRO gene product, which is a repressor exercising a negative mode of control. Conversely, during lysogenic growth (cell type B in Table II), the lytic functions (A-specific functions) are in low demand and are predicted to involve the negative mode of control. Indeed, they are controlled by the CI gene product, which is a repressor exercising a negative mode of control. At the same time, the lysogenic functions (B-specific func-
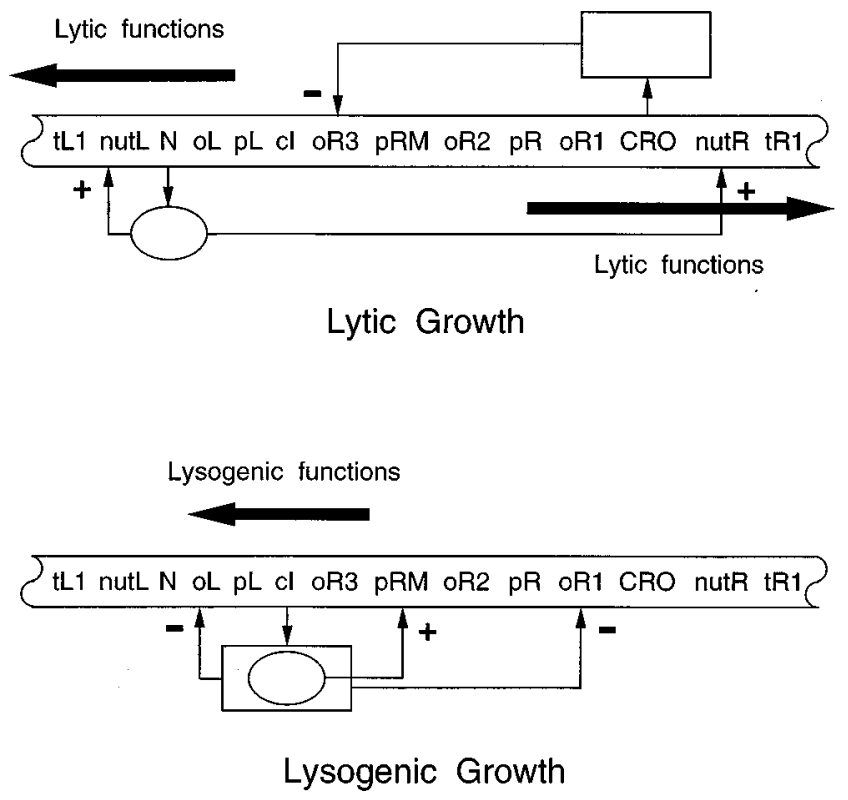

FIG. 7. Switching the mode of control for regulated cell-specific functions during differentiation. The temperate bacteriophage $\lambda$ can be considered a simple model system that exhibits two differentiated forms: (Top panel) The lytic form in which the phage infects a cell, multiplies to produce multiple phage copies, lysis the cell, and the released progeny begin another cycle of lytic growth. (Bottom panel) The lysogenic form in which the phage genome is stably incorporated into the host cell DNA and is replicated passively once each time the host genome is duplicated. During differentiation, when the lysogenic phage is induced to become a lytic phage or the lytic phage becomes a lysogenic phage upon infection of a bacterial cell, the mode of control switches from positive to negative or vice versa because of the interlocking gene circuitry of phage $\lambda$. See text for further discussion.

tions) are in high demand and are predicted to involve the positive mode of control. In this case, they are controlled by the CI gene product, which is also an activator exercising a positive mode of control. The mode in each individual case is predicted correctly, and the switching of modes during "differentiation" (from lysogenic to lytic growth or vice versa) is brought about by the interlocking circuitry of phage $\lambda$.

\section{B. Coupling of elementary gene circuits}

There are logically just three patterns of coupling between the expression cascades for regulator and effector proteins in elementary gene circuits. These are the directly coupled, uncoupled, and inversely coupled patterns in which regulator gene expression increases, remains unchanged, or decreases with an increase in effector gene expression (Fig. 8). Elementary gene circuits in bacteria have long been studied and there are well-characterized examples that exhibit each of these patterns.

A design principle governing the pattern of coupling in such circuits has been identified by mathematically controlled comparison of various designs. ${ }^{11}$ The principle is expressed in terms on two properties: the mode of control (positive or negative) and the capacity for regulated expression (large or small ratio of maximal to basal level of expression). According to this principle, one predicts that elementary gene circuits with the negative mode and small, intermediate, and large capacities for gene regulation will 


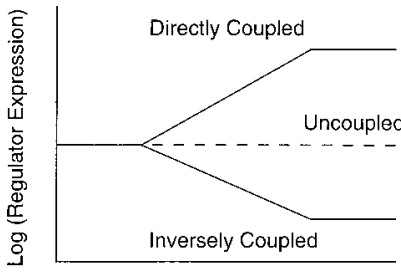

$\log$ (Stimulus)

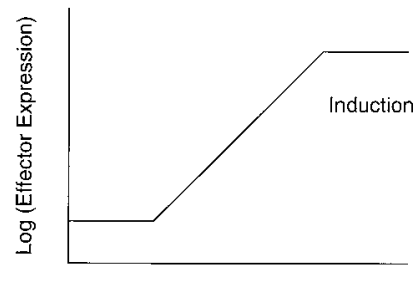

$\log$ (Stimulus)
FIG. 8. Coupling of expression in elementary gene circuits. The panel on the right shows the steady-state expression characteristic for the effector cascade in Fig. 6. The panel on the left shows the steady-state expression characteristic for the regulator cascade. Induction of effector expression occurs while regulator expression increases (directly coupled expression), remains unchanged (uncoupled expression), or decreases (inversely coupled expression).

exhibit direct coupling, uncoupling, and inverse coupling, respectively. Circuits with the positive mode, in contrast, are predicted to have inverse coupling, uncoupling, and direct coupling.

The approach used to identify this design principle involves (1) formulating kinetic models that are sufficiently generic to include all of the logical possibilities for coupling of expression in elementary gene circuits, (2) making these models equivalent in all respects other than their regulatory parameters, (3) identifying a set of a priori criteria for functional effectiveness of such circuits, (4) analyzing the steadystate and dynamic behavior of the various designs, and (5) comparing the results to determine which designs are better according to the criteria. These steps are outlined next.

The kinetic models are all special cases of the generic model that is graphically depicted in Fig. 6. This model, which captures the essential features of many actual circuits, includes two transcription units: one for a regulator gene and another for a set of effector genes. The regulator gene encodes a protein that acts at the level of transcription to bring about induction, and the effector genes encode the enzymes that catalyzes a pathway of reactions in which the inducer is an intermediate. The regulator can negatively or positively influence transcription at the promoter of each transcription unit, and these influences, whether negative or positive, can be facilitated or antagonized by the inducer. A local powerlaw representation that describes the regulatable region (i.e., the inclined portion) of the steady-state expression characteristics in Fig. 8 is the following:

$$
\begin{aligned}
& d X_{1} / d t=\alpha_{1} X_{3}^{g^{13}} X_{5}^{{ }^{g} 15} X_{6}^{g^{16}}-\beta_{1} X_{1}^{h_{11}}, \\
& d X_{2} / d t=\alpha_{2} X_{1}^{{ }^{g}}{ }^{21} X_{7}^{g_{27}}-\beta_{2} X_{2}^{h_{22}}, \\
& d X_{3} / d t=\alpha_{3} X_{2}^{{ }^{g}}{ }^{32} X_{8}{ }^{g_{38}}-\beta_{3} X_{2}^{h_{32}} X_{3}^{h_{33}} \text {, } \\
& d X_{4} / d t=\alpha_{4} X_{3}^{{ }^{g}}{ }_{43} X_{5}{ }^{g_{45}} X_{6}{ }^{g_{46}}-\beta_{4} X_{4}{ }^{h_{44}} \text {, } \\
& d X_{5} / d t=\alpha_{5} X_{4}^{g_{54}} X_{7}^{g_{57}}-\beta_{5} X_{5}{ }^{h_{55}} .
\end{aligned}
$$

There are four parameters that characterize the pattern of regulatory interactions: $g_{13}$ and $g_{43}$ quantify influences of inducer $X_{3}$ on the rate of synthesis of effector mRNA $X_{1}$ and regulator mRNA $X_{4}$, whereas $g_{15}$ and $g_{45}$ quantify influences of regulator $X_{5}$ on these same processes.
TABLE III. Predicted patterns of coupling for regulator and effector cascades in elementary gene circuits.

\begin{tabular}{ccl}
\hline \hline Mode of control & Capacity for regulation $^{\mathrm{a}}$ & Pattern of coupling \\
\hline Positive & Large & Directly coupled \\
Positive & Intermediate & Uncoupled \\
Positive & Small & Inversely coupled \\
Negative & Large & Inversely coupled \\
Negative & Intermediate & Uncoupled \\
Negative & Small & Directly coupled \\
\hline \hline
\end{tabular}

${ }^{a}$ Capacity for regulation is defined as the ratio of maximal to basal level of expression.

In the various models, the values for all corresponding parameters other than the four regulatory parameters are made equal (internal equivalence). The four regulatory parameters have their values constrained so as to produce the same steady-state expression characteristics (external equivalence). Models exhibiting each of the three patterns of coupling are represented within the space of the constrained regulatory parameters.

Six quantitative, a priori criteria for functional effectiveness are used as a basis for comparing the behavior of the various models. These are decisiveness, efficiency, selectivity, stability, robustness, and responsiveness. A decisive system has a sharp threshold for response to substrate. An efficient system makes a large amount of product from a given supra-threshold increment in substrate. A selective system governs the amount of regulator so as to ensure specific control of effector gene expression. A locally stable system returns to its original state following a small perturbation. A robust system tends to maintain its state despite changes in parameter values that determine its structure. A responsive system quickly adjusts to changes. (Further discussion of these criteria and the means by which they are quantified can be found elsewhere. ${ }^{11}$ )

The steady-state and dynamic behavior of the various models is analyzed by standard algebraic and numerical methods, and the results are quantified according to the above criteria. Temporal responsiveness is a distinguishing criterion for effectiveness of these circuits. A comparison of results for models with the various patterns of coupling leads to the predicted correlations summarized in Table III.

To test these predicted correlations we identified 32 elementary gene circuits for which the mode of control was known and for which quantitative data regarding the capacities for regulator and effector gene expression were available in the literature. A plot of these data in Fig. 9 shows reasonable agreement with the predicted positive slope for the points representing circuits with a positive mode and the predicted negative slope for the points representing circuits with a negative mode. Global experiments that utilize microarray technology could provide more numerous and potentially more accurate tests of these predictions.

\section{Connectivity and switching}

Gene expression can be switched ON (and OFF) in either a discontinuous dynamic fashion or a continuously variable static fashion in response to developmental or environ- 


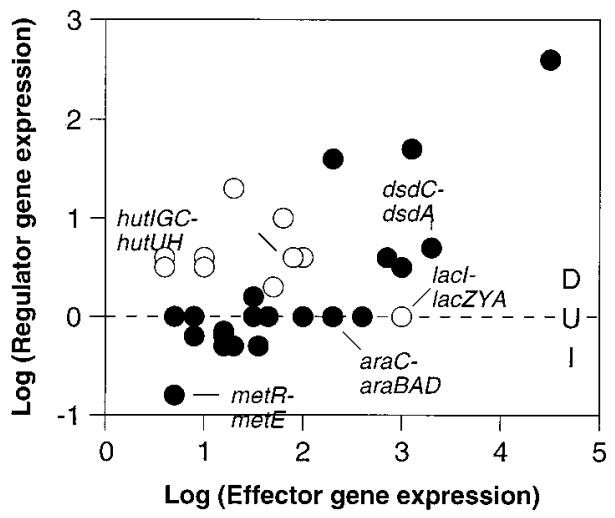

FIG. 9. Experimental data for the coupling of expression in elementary gene circuits. The capacity for induction of the effector cascade is plotted on the horizontal axis as positive values. The capacity for expression of the regulator cascade is plotted on the vertical axis as positive values (induction), negative values (repression), or zero (no change in expression). Effector cascades having a positive mode of control are represented as data points with filled symbols and those having a negative mode with open symbols. Data show reasonably good agreement with the predictions in Table III.

mental cues. These alternative switch behaviors are clearly manifested in the steady-state expression characteristic of the gene. In some cases, the elements of the circuitry appear to be the same, and yet the alternative behaviors can be gener- ated by the way in which the elements are interconnected. This design feature has been examined in simple model circuits. The results have led to specific conditions that allow one to distinguish between these alternatives, and these conditions can be used to interpret the results of experiments with the lac operon of E. coli.

A design principle that distinguishes between discontinuous and continuous switches in a model for inducible catabolic pathways (Fig. 10) is the following. If the natural inducer is the initial substrate of the inducible pathway, or if it is an intermediate in the inducible pathway, then the switch will be continuous; if the inducer is the final product of the inducible pathway, then the switch can be discontinuous or continuous, depending on an algebraic condition that involves four kinetic orders for reactions in the circuit. (A more general statement of the principle can be given in terms of the algebraic condition, as will be shown below.)

A simplified set of equations that captures the essential features of the model in Fig. 10 is the following:

$$
\begin{aligned}
& d X_{1} / d t=\alpha_{1 B}-\beta_{1} X_{1}, \quad X_{3}<X_{3 L}, \\
& d X_{1} / d t=\alpha_{1} X_{3}^{{ }^{g} 13}-\beta_{1} X_{1}, \quad X_{3 L}<X_{3}<X_{3 H}, \\
& d X_{1} / d t=\alpha_{1 M}-\beta_{1} X_{1}, \quad X_{3 H}<X_{3},
\end{aligned}
$$

A

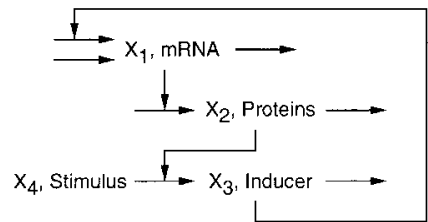

D

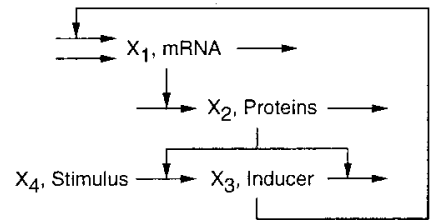

G

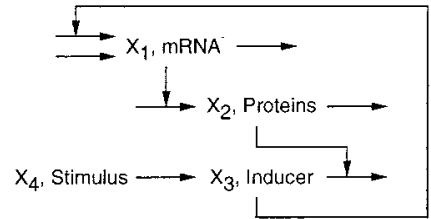

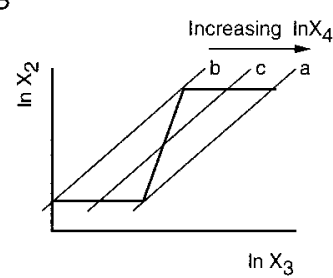

$E$

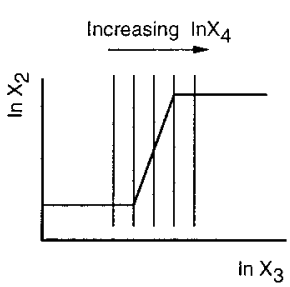

$\mathrm{H}$

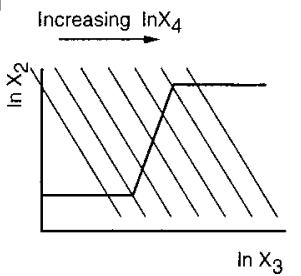

C

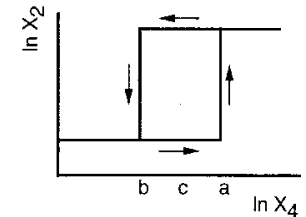

F
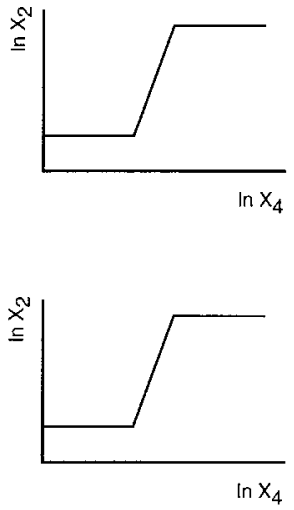

FIG. 10. Simplified model of an inducible catabolic pathway exhibits two types of switch behavior depending upon the position of the inducer in the inducible pathway. (a) The inducer is the final product of the inducible pathway. (b) The S-shaped curve is the steady-state solution for Eqs. (9) and (10). The lines (a, $b$, and $c$ ) are the steady-state solutions for Eq. (11) with different fixed concentrations of the stimulus $X_{4}$. The steady-state solutions for the system are given by the intersections of the S-shaped curve and the straight lines. There is only one intersection (maximal expression) when $\ln X_{4}>a$; there is only one intersection (basal expression) when $\ln X_{4}<b$. There are three intersections when $b<\ln X_{4}=c<a$, but the middle one is unstable. The necessary and sufficient condition for the bistable behavior in this context is that the slope of the straight line be less than the slope of the S-shaped curve at intermediate concentrations of the inducer $X_{3}$, which is the condition expressed in Eq. (12). (c) The steady-state induction characteristic exhibits discontinuous dynamic switches and a well-defined hysteresis loop. Thus, at intermediate concentrations of the stimulus $X_{4}$, expression will be at either the maximal or the basal level depending upon the past history of induction. (d) The inducer is an intermediate in the inducible pathway. (e) The steady-state solutions for the system are given by the intersections of the S-shaped curve and vertical lines. There is only one intersection possible for any given concentration of stimulus. (f) The steady-state induction characteristic exhibits a continuously changing static switch. (g) The inducer is the initial substrate of the inducible pathway. (h) The steady-state solutions for the system are given by the intersections of the S-shaped curve and the lines of negative slope. There is only one intersection possible for any given concentration of stimulus. (i) The steady-state induction characteristic exhibits a continuously changing static switch. 
TABLE IV. Summary of predictions relating type of switch behavior to the connectivity in the model inducible circuit of Fig. 10.

\begin{tabular}{llllll}
\hline \hline Figure & Stimulus & Inducer & Transport & $\begin{array}{l}\text { Connection from } \\
\text { inducible pathway }\end{array}$ & Switch \\
\hline $10(\mathrm{~d})$ & IPTG & IPTG & Constitutive & None & Static \\
$10(\mathrm{a})$ & IPTG & IPTG & Inducible & Product & Dynamic \\
$10(\mathrm{~d})$ & Lactose & Allolactose & Inducible & Intermediate & Static \\
$10(\mathrm{~g})$ & Allolactose & Allolactose & Constitutive & Substrate & Static \\
\hline \hline
\end{tabular}

$$
\begin{aligned}
& d X_{2} / d t=\alpha_{2} X_{1}-\beta_{2} X_{2}, \\
& d X_{3} / d t=\alpha_{3} X_{2}^{g_{32}} X_{4}^{g_{34}}-\beta_{3} X_{2}^{h_{32}} X_{3}^{h_{33}} .
\end{aligned}
$$

The variables $X_{1}, X_{2}, X_{3}$, and $X_{4}$ represent the concentrations of polycistronic mRNA, a coordinately regulated set of proteins, inducer, and stimulus, respectively. This is a piecewise power-law representation (see Appendix of Ref. 27) that emphasizes distinct regions of operation. There is a constant basal level of expression when inducer concentration $X_{3}$ is lower than a value $X_{3 L}$; there is a constant maximal level of expression when inducer concentration is higher than a value $X_{3 H}$; there is a regulated level of expression (with cooperativity indicated by a value of the parameter $\left.g_{13}>1\right)$ when inducer concentration is between the values $X_{3 L}$ and $X_{3 H}$. All parameters in this model have positive values.

The position of the natural inducer in an inducible pathway has long been known to have a profound effect on the local stability of the steady state when the system is operating on the inclined portion (i.e., the regulatable region) of the steady-state expression characteristic (Fig. 8, right panel). ${ }^{27}$ As the position of the natural inducer is changed from the initial substrate [Fig. 10(g)], to an intermediate [Fig. 10(d)] to the final product [Fig. 10(a)] of the inducible pathway (all other parameters having fixed values), the margin of stability decreases. In this progression the single stable steady state [Fig. 10(h)] can undergo a bifurcation to an unstable steady state flanked by two stable steady states [Fig. 10(b)], which is the well-known cusp catastrophe characteristic of a dynamic ON-OFF switch. ${ }^{41}$

The critical conditions for the existence of multiple steady states and a dynamic switch are given by

$$
g_{13}>h_{33} /\left(g_{32}-h_{32}\right) \text { and } g_{32}>h_{32} \text {. }
$$

In general, the inducible proteins must have a greater influence on the synthesis $\left(g_{32}\right)$ than on the degradation $\left(h_{32}\right)$ of the inducer. These conditions can be interpreted, according to conventional assumptions, in terms of inducer position in the pathway. If the position of the true inducer is functionally equivalent to that of the substrate for the inducible pathway, then $g_{32}=0$ and the conditions in Eq. (12) cannot be satisfied. If the position is functionally equivalent to that of the intermediate in the inducible pathway, the kinetic orders for the rates of synthesis and degradation of the intermediate are the same with respect to the enzymes for synthesis and degradation, and these enzymes are coordinately induced, then $g_{32}=h_{32}$ and again the conditions in Eq. (12) cannot be satisfied. However, if the position is functionally equivalent to that of the product for the inducible pathway, then $h_{32}=0$ and the conditions in Eq. (12) can be satisfied provided $g_{13}$ $>h_{33} / g_{32}$.

The values of the parameters in this model have been estimated from experimental data for the lac operon of $E$. coli. ${ }^{10}$ These results, together with these data, can be used to interpret four experiments involving the circuitry of the lac operon (see Table IV and the following discussion).

First, if the lac operon is induced with the nonmetabolizable (gratuitous) inducer isopropyl- $\beta$, D-thiogalactoside (IPTG) in a cell with the inducible Lac permease protein, then the model is as shown in Fig. 10(a). In this case, $X_{1}$ is the concentration of polycistronic lac mRNA, $X_{2}$ is the concentration of the Lac permease protein alone $\left(X_{2}\right.$ has no influence on the degradation of the inducer $\left.X_{3}\right), X_{3}$ is the intracellular concentration of IPTG, and $X_{4}$ is the extracellular concentration of IPTG. With the parameter values from the lac operon, the conditions in Eq. (12) are satisfied because $h_{33}=1$ (aggregate loss by all causes in exponentially growing cells is first order), $g_{32}=1$ (enzymatic rate is first order with respect to the concentration of total enzyme), and $g_{13}=2$ (the Hill coefficient of lac transcription with respect to the concentration of inducer is second order).

Second, if the lac operon is induced with the gratuitous inducer IPTG in a cell without the Lac permease protein, then the inducer IPTG is not acted upon by any of the protein products of the operon. In this case, $X_{1}$ is the concentration of polycistronic lac mRNA, $X_{2}$ is the concentration of $\beta$-galactosidase protein alone ( $X_{2}$ has no influence on either the synthesis or the degradation of the inducer $\left.X_{3}\right), X_{3}$ is the intracellular concentration of IPTG, and $X_{4}$ is the extracellular concentration of IPTG. The conditions in Eq. (12) now cannot be satisfied because $g_{23}=h_{23}=0$ and all other parameters are positive. This is an open-loop situation in which expression of the operon is simply proportional to the rate of transcription as determined by the steady-state concentration of intracellular IPTG, which is proportional to the concentration of extracellular IPTG.

Thus, the kinetic model accounts for two important observations from previous experiments. It accounts for the classic experimental results of Novick and Weiner ${ }^{42}$ in which they observed a discontinuous dynamic switch with hysteresis. They induced the lac operon with a gratuitous inducer that was transported into the cell by the inducible Lac permease, was diluted by cellular growth, but was not acted upon by the remainder of the inducible pathway. Hence, the gratuitous inducer occupied the position of final product for the inducible pathway (in this case simply the Lac permease 
step), and the model predicts dynamic bistable switch behavior similar to that depicted in Figs. 10(b) and 10(c). The kinetic model also accounts for the classic experimental results of Sadler and Novick ${ }^{43}$ in which they observed a continuous static switch without hysteresis. In their experiments they used a mutant strain of E. coli in which the lac permease was inactivated and they induced the lac operon with a gratuitous inducer. In this system, the inducer is not acted upon by any part of the inducible pathway, the extracellular and intracellular concentrations of inducer are proportional, and the model predicts a continuous static switch similar to that depicted in Figs. 10(e) and 10(f). The model in Fig. 10 also makes two other predictions related to the position of the natural inducer in the inducible pathway.

First, if the lac operon is induced with lactose in a cell with all the inducible Lac proteins intact, then the model is as shown in Fig. 10(d). In this case, $X_{1}$ is the concentration of polycistronic lac mRNA, $X_{2}$ is the concentration of the Lac permease protein as well as the concentration of the $\beta$-galactosidase protein (which catalyzes both the conversion of lactose to allolactose and the conversion of allolactose to galactose and glucose), $X_{3}$ is the intracellular concentration of allolactose, and $X_{4}$ is the extracellular concentration of lactose. In steady state, the sequential conversion of extracellular lactose to intracellular lactose (by Lac permease) and intracellular lactose to allolactose (by $\beta$-galactosidase) can be represented without loss of generality as a single process because these two proteins are coordinately expressed. Again, the conditions in Eq. (12) cannot be satisfied. In this case, $g_{23}=h_{23}=1$ and all other parameters are positive finite, and the model predicts a continuous static switch similar to that depicted in Figs. 10(e) and 10(f).

Second, if the lac operon is induced with allolactose, the natural inducer, in a cell without the Lac permease protein, then the model is as shown in Fig. $10(\mathrm{~g})$. In this case, $X_{1}$ is the concentration of polycistronic lac mRNA, $X_{2}$ is the concentration of the $\beta$-galactosidase protein alone (which catalyzes the conversion of allolactose to galactose and glucose), $X_{3}$ is the intracellular concentration of allolactose, and $X_{4}$ is the extracellular concentration of allolactose. The conditions in Eq. (12) cannot be satisfied. In this case, $g_{23}=0$ and all other parameters are positive, and the model predicts a continuous static switch similar to that represented in Figs. 10(h) and $10(\mathrm{i})$.

The fact that the kinetic model of the lac operon predicts a continuous static switch in response to extracellular lactose led us to search the literature for the relevant experimental data. We were unable to find any experimental evidence for either a continuous static switch or a discontinuous dynamic switch in response to lactose, which comes as a surprise. Despite the long history of study involving the lac operon, such experiments apparently have not been reported. Experiments to test this prediction specifically are currently being designed and carried out (Atkinson and Ninfa, unpublished results).

\section{Context and logic}

In the qualitative version of demand theory (Sec. IV A) it was assumed for simplicity that there was a constant demand regime for the effector gene in question and that its expression was controlled by a single regulator. Here I review the quantitative version of demand theory and include consideration of genes exposed to more than one demand regime and controlled by more than one regulator.

\section{Life cycle provides the context for gene control}

Models that include consideration of the organism's life cycle, molecular mode of gene control, and population dynamics are used to describe mutant and wild-type populations in two environments with different demands for expression of the genes in question. These models are analyzed mathematically in order to identify conditions that lead to either selection or loss of a given mode of control. It will be shown that this theory ties together a number of important variables, including growth rates, mutation rates, minimum and maximum demands for gene expression, and minimum and maximum durations for the life cycle of the organism. A test of the theory is provided by the lac operon of E. coli.

The life cycle of $E$. coli involves sequential colonization of new host organisms, ${ }^{44}$ which means repeated cycling between two different environments [Figs. 11(a) and 11(b)]. In one, the upper portion of the host's intestinal track, the microbe is exposed to the substrate lactose and there is a high demand for expression of the lac operon, and in the other, the lower portion of the intestinal track and the environment external to the host, the microbe is not exposed to lactose and there is a low demand for lac expression. The average time to complete a cycle through these two environments is defined as the cycle time, $C$, and the average fraction of the cycle time spent in the high-demand environment is defined as the demand for gene expression, $D$ [Fig. 11(c)].

The implications for gene expression of mutant and wild-type operons in the high- and low-demand environments are as follows. The wild-type functions by turning on expression in the high-demand environment and turning off expression in the low-demand environment. The mutant with a defective promoter is unable to turn on expression in either environment. The mutant with a defective modulator (or defective regulator protein) is unable to turn off expression regardless of the environment. The double mutant with defects in both promoter and modulator/regulator behaves like the promoter mutant and is unable to turn on expression in either environment.

The sizes of the populations are affected by the transfer rate between populations, which is the result of mutation, and by the growth rate, which is the result of overall fitness. The transfer rates depend on the mutation rate per base and on the size of the relevant target sequence. The growth rate for the wild type is greater than that for mutants of the modulator type in the low-demand environment; these mutants are selected against because of their superfluous expression of an unneeded function. The growth rate for the wild type is greater than that for mutants of the promoter type in the high-demand environment; these mutants are selected against because of their inability to express the needed function.

Solution of the dynamic equations for the populations cycling through the two environments yields expressions in 
A

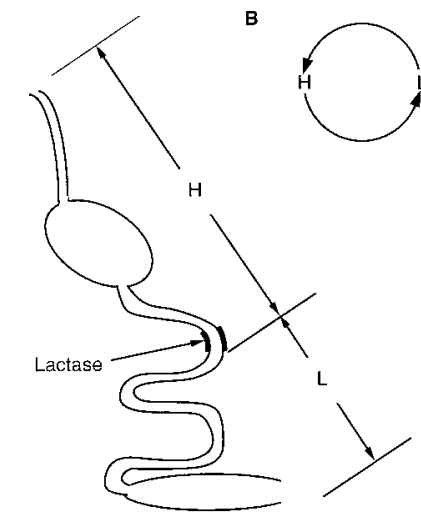

C

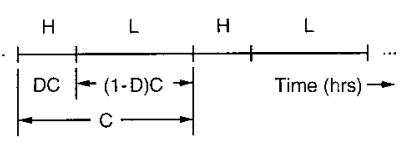

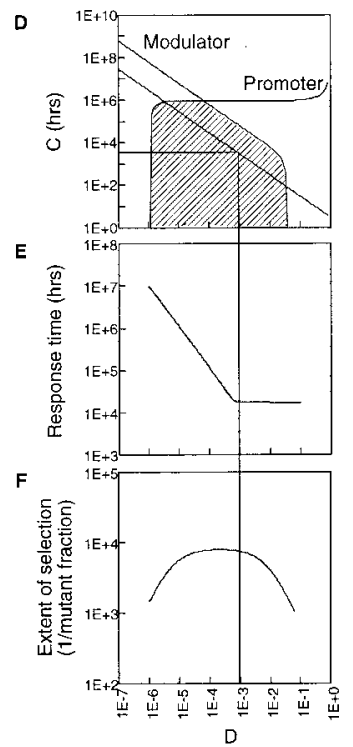

FIG. 11. Life cycle of Escherichia coli and the demand for expression of its lac operon. (a) Schematic diagram of the upper (high demand) and lower (low demand) portions of the human intestinal track. (b) Life cycle consists of repeated passage between environments with high- and low-demand for lac gene expression. (c) Definition of cycle time $C$ and demand for gene expression $D$. (d) Region in the $C$ vs $D$ plot for which selection of the wild-type control mechanism is possible. (e) Rate of selection as a function of demand. (f) Extent of selection as a function of demand. See text for discussion.

$C$ and $D$ for the threshold, extent, and rate of selection that apply to the wild-type control mechanism. ${ }^{45}$ The threshold for selection is given by the boundary of the shaded region in Fig. 11(d); only systems with values of $C$ and $D$ that fall within this region are capable of being selected. The rate and extent of selection shown in Figs. 11(e) and 11(f) exhibit optimum values for a specific value of $D$.

Application of this quantitative demand theory to the lac operon of E. coli yields several new and provocative predictions that relate genotype to phenotype. ${ }^{46}$ The straight line in Fig. 11(d) represents the inverse relationship $C=3 / D$ that results from fixing the time of exposure to lactose at $3 \mathrm{~h}$, which is the clinically determined value for humans. ${ }^{47,48}$ The intersections of this line with the two thresholds for selection provide lower and upper bounds on the cycle time. The lower bound is approximately $24 \mathrm{~h}$, which is about as fast as the microbe can cycle through the intestinal track without colonization. $^{49-51}$ The upper bound is approximately 70 years, which is the longest period of colonization without cycling and corresponds favorably with the maximum life span of the host. ${ }^{52}$ The optimum value for the cycle time, as determined by the optimum value for demand [from Figs. 11(e) and 11(f)], is approximately four months, which is comparable to the average rate of recolonization measured in humans. ${ }^{53-55}$ A summary of these results is given in Table $\mathrm{V}$.

\section{Logic unit and phasing of lac control}

The analysis in Sec. IV D 1 assumed that when E. coli was growing on lactose there was no other more preferred carbon source present. Thus, the positive CAP-cAMP regulator ${ }^{56}$ was always present, and we could then concen-

TABLE V. Summary of experimental data and model predictions based on conditions for selection of the lac operon in Escherichia coli.

\begin{tabular}{lcc}
\hline \hline Characteristic & Experimental data & Model predictions \\
\hline Intestinal transit time & $12-48 \mathrm{~h}$ & $26 \mathrm{~h}$ \\
Lifetime of the host & 120 years & 66 years \\
Re-colonization rate & $2-18$ months & 4 months \\
\hline
\end{tabular}

trate on the conditions for selection of the specific control by Lac repressor. This was a simplifying assumption; in the more general situation, both the specific control by Lac repressor and the global control by CAP-cAMP activator must be taken into consideration. The analysis becomes more complex, but it follows closely the outline of the simpler case in Sec. IV D 1.

By extension of the definition for demand $D$, given in Sec. IV D 1, one can define a period of demand for the absence of repressor $G$, a period of demand for the presence of activator $E$, and a phase relationship between these two periods of demand $F$. By extension of the analysis in Sec. IV D 1, solution of the dynamic equations for wild-type and mutant populations cycling through the two environments yields expressions in $C, G, E$, and $F$ for the threshold, extent, and rate of selection that apply to the wild-type control mechanism.

The threshold for selection is now an envelope surrounding a "mound" in four-dimensional space with cycle time $C$ as a function of the three parameters $G, E$, and $F$; only systems with values that fall within this envelope are capable of being selected. The rate and extent of selection exhibit optimum values as before, but these now occur with a specific combination of values for $G, E$, and $F$. The values of $G, E$, and $F$ that yield the optima represent a small period when repressor is absent, an even smaller period when activator is absent, and a large phase period between them. The period when repressor is absent corresponds to the period of exposure to lactose $(\sim 0.36 \%$ of the cycle time). Within this period (but shifted by $\sim 0.20 \%$ of the cycle time) there is a shorter period when activator is absent $(\sim 0.14 \%$ of the cycle time); this corresponds to the presence of a more preferred carbon source that lowers the level of cAMP.

These relationships can be interpreted in terms of exposure to lactose, exposure to glucose, and expression of the lac operon as shown in Fig. 12. As E. coli enters a new host, passes through the early part of the intestinal track, and is exposed to lactose, the lac operon is induced and the bacteria are able to utilize lactose as a carbon source. During this period the operator site of the lac operon is free of the Lac repressor. At the point in the small intestine where the host's lactase enzymes are localized, lactose is actively split into its constituent sugars, glucose and galactose. This creates a rapid elevation in the concentration of these sugars in the environment of E. coli. A period of growth on glucose is initiated, and this is accompanied by catabolite repression and lactose exclusion from the bacteria. During this period the initiator site of the lac operon is free of the CAP-cAMP activator, transcription of the lac operon ceases, and the concentration of $\beta$-galactosidase is diluted by growth. During 


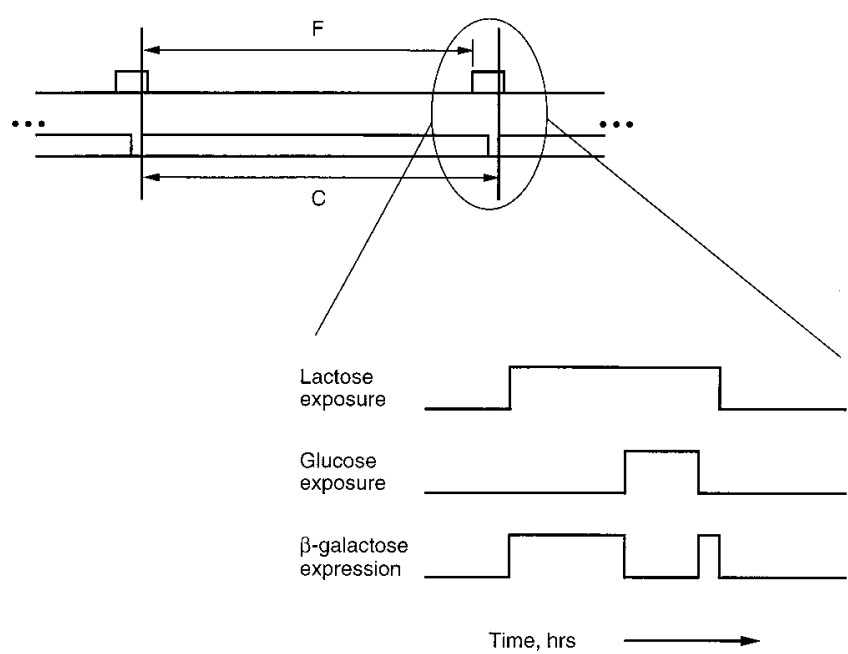

FIG. 12. Optimal duration and phasing of the action by the positive (CAPcAMP) and negative (LacI) regulators of $\beta$-galactosidase expression. The signal on the top line represents the absence of repressor binding to the lac operator site, the signal on the second line represents activator binding to the lac initiator site. The cycle time $C$ is the period between the vertical lines, and the relative phasing is shown as $F$. An expanded view of the critical region gives an interpretation in terms of exposure to lactose and glucose as bacteria pass the site of the lactase enzymes in the small intestine. See text for discussion.

this period the glucose in the intestine is also rapidly absorbed by the host. Eventually, the glucose is exhausted, the CAP-cAMP activator again binds the initiator site of the lac operon, and the residual lactose that escaped hydrolysis by the host's lactase enzymes causes a diminished secondary induction of the bacterial lac operon. Finally, the lactose is exhausted, the Lac repressor again binds the operator site of the lac operon, and the microbe enters the low-demand environment and colonizes the host.

The quantitative version of demand theory integrates information at the level of DNA (mutation rate, effective target sizes for mutation of regulatory proteins, promoter sites, and modulator sites), physiology (selection coefficients for superfluous expression of an unneeded function and for lack of expression of an essential function), and ecology (environmental context and life cycle) and makes rather surprising predictions connected to the intestinal physiology, life span, and recolonization rate of the host. There is independent experimental data to support each of these predictions.

Finally, when the logic of combined control by CAPcAMP activator and Lac repressor was analyzed, we found an optimum set of values not only for the exposure to lactose, but also for the exposure to glucose and for the relative phasing between these periods of exposure. The phasing predicted is consistent with the spatial and temporal environment created by the patterns of disaccharide hydrolysis and monosaccharide absorption along the intestinal tract of the host.

\section{DISCUSSION}

Although biological principles that govern some variations in design have been identified (e.g., positive vs negative modes of control), there are other well-documented (and many not so well-documented) variations in design that still are not understood. For example, why is the positive mode of control in some cases realized with an activator protein that facilitates transcription of genes downstream of a promoter, and in other cases with an antiterminator protein that facilitates transcription of genes downstream of a terminator? There are many examples of each, but no convincing explanation for the difference. Thus, the elements of design and the variations I have reviewed in Sec. II provide only a start; there is much to be done in this area.

For the comparative analysis of alternative designs we require a formalism capable of representing diverse designs, tractable methods of analysis for characterizing designs, and a strategy for making well-controlled comparisons that reveal essential differences while minimizing extraneous differences. As reviewed in Sec. III, there are several arguments that favor the power-law formalism for representing a wide spectrum of nonlinear systems. In particular, the local S-system representation within this formalism not only provides reasonably accurate descriptions but also possesses a tractable structure, which allows explicit solutions for the steady state and efficient numerical solutions for the dynamics. Explicit steady-state solutions are used to make mathematically controlled comparisons. Constraining these solutions provides invariants that eliminate extra degrees of freedom, which otherwise would introduce extraneous differences into the comparison of alternatives. The ability to provide such invariants is one of the principle advantages of using the local S-system representation. Two other formalisms with this property are the linear representation and the Volterra-Lotka representation, which is equivalent to the linear representation for the steady state. However, these representations yield linear relations between variables in steady state, which is less appropriate for biological systems in which these relationships are typically nonlinear.

The utility of these methods for studying alternative designs ultimately will be determined by the degree to which their predictions are supported by experimental evidence. For this reason it is important that the methods consider an entire class of systems without specifying numerical values for the parameters, which often are unknown in any case. Predictions achieved with this approach then can be tested against numerous examples provided by members of the class. If the methods were to focus upon a single system with specific values for its parameters, then there would be only the one example to test any hypothesis that might be conceived. The symbolic approach also allows one to compare efficiently many alternatives including ones that no longer exist (and so values of their parameters will never be known), which often is the case in trying to account for the evolution of a given design, or that hypothetically might be brought into existence through genetic engineering. The four design principles reviewed in Sec. IV illustrate the types of results that have been obtained when the methods in Sec. III are applied to some of the elements of design described in Sec. II.

First, we examined the two modes of control in elementary gene circuits (Sec. IV A). Qualitative arguments and examples were used to demonstrate the validity of demand 
theory for the regulator-modulator component of control mechanisms. The same approach also can be used to account for the alternative forms of the promoter component. In either case, the qualitative arguments are based on extreme cases where the demand is clearly high or low. One would like to quantify what is meant by demand, to know how high it must be to select for the positive mode of control or a low-level promoter, and to know how low it must be to select for the negative mode of control or a high-level promoter. The quantitative version of demand theory reviewed in Sec. IV D specifically addresses these issues.

Second, we examined the three patterns of coupling in elementary gene circuits (Sec. IV B). It was their dynamic properties that proved to be distinctive. Establishing the dynamic differences required efficient numerical solutions of the differential equations and a means to reduce the dimension of the search in order to explore fully the parameter space. The results in Sec. IV B illuminate an area of experimental work that needs greater attention. For example, the data in Fig. 9 were obtained from individual gene circuits as a result of labor-intensive studies designed for purposes other than quantitative characterization of the steady-state induction characteristics for effector and regulator cascades. The data often are sketchy and subject to large errors, particularly in the case of regulator proteins, which generally are expressed at very low levels. Genomic and proteomic approaches to the measurement of expression should provide data for a much larger number of elementary gene circuits. However, these approaches also have difficulty measuring low levels of expression, and so technical improvements will be needed before they will be able to quantify expression of regulator genes.

Third, we examined various forms of connectivity that link the inducer to the transcription unit for an inducible catabolic pathway and showed that two different types of switching behavior result (Sec. IV C). The analysis of lac circuitry in this regard focused attention on a long-standing misconception in the literature, namely, that lac operon expression normally is an all-or-none phenomenon. While continuously variable induction of the lactose operon might be appropriate for a catabolic pathway whose expression can provide benefits to the cell even when partially induced, a discontinuous induction with hysteresis might be more appropriate for major differentiation events that require a definite commitment at some point. The wider the hysteretic loop the greater the degree of commitment. The width of the loop tends to increase with a large capacity for induction (ratio of maximum to basal level of expression), high logarithmic gain in the regulatable region (high degree of cooperativity), and substrates for the enzymes in the pathway operating as near saturation as compatible with switching.

Fourth, we examined the context of gene expression and developed a quantitative version of demand theory (Sec. IV D). In addition to providing a quantitative measure of demand, the results define what high and low mean in terms of the level of demand required to select for the positive or the negative mode of control and for low- or high-level promoters. This analysis also predicted new and unexpected kinds of information, such as intestinal transit time, host life- time, and recolonization rate. When the logic unit involving the two relevant regulators was included in the analysis it also yielded predictions for the relative phasing of the environmental cues involved in lac operon induction.

Is there anything common to these successful explanations of design that might be useful as a guide in exploring other variations in design? Two such features come to mind. First, each of the examples involved a limited number of possible variations on a theme: two modes of control, three patterns of coupling, two types of switches. This meant that only a small number of cases had to be analyzed and compared, which is a manageable task. If there had been many variations in each case, then one would have no hope of finding a simple underlying rule that could account for all the variations, and one might never have considered analyzing and comparing all of the possibilities. Second, each case could be represented by a set of simple equations whose structure allowed symbolic analysis (and exhaustive numerical analysis when necessary). This permitted the use of controlled mathematical comparisons, which led to the identification of clear qualitative differences in the behavior of the alternatives. Thus, it might prove fruitful in the future to look for instances where these features present themselves.

In this context, we must acknowledge the fundamental role of accident in generating the diversity that is the substrate for natural selection. Thus, there undoubtedly will be examples of recently generated variations in design for which there will be no rational explanation. Only in time will natural selection tend to produce designs that are shaped for specific functions and hence understandable in principle.

Finally, will the understanding of large gene networks require additional tools beyond those needed for elementary gene circuits? Although we have no general answer to this question, there are three points having to do with network connectivity, catalytic versus stoichiometric linkages, and time-scale separation that are worthy of comment.

First, the evidence suggests, at least for bacteria, that there are relatively few connections between elementary gene circuits (see Sec. IID). This probably explains the experimental success that has been obtained by studying the regulation of isolated gene systems. Had there been rich interactions among these gene systems, such studies might have been less fruitful. Low connectivity also suggests that the understanding of elementary circuits may largely carry over to their role in larger networks and that the same tools might be used to study larger networks.

Second, catalytic linkages between circuits are less problematic then stoichiometric linkages, at least for the analysis of steady-state behavior. Elementary circuits can be linked catalytically without their individual properties changing appreciably, because the molecules in one circuit acting catalytically on another circuit are not consumed in the process of interaction. Such a circuit can have a unilateral effect on a second circuit, without having its own behavior affected in the process. This permits a modular block-diagram treatment, which makes use of the results obtained for the individual circuits in isolation, to characterize the larger network. (This is analogous to the well-known strategy used by electronic engineers, who design operational amplifiers with 
high impedance to insulate the properties of the modules being coupled.) On the other hand, elementary circuits that are linked stoichiometrically may not be treatable in this fashion, because the molecules in one circuit are consumed in the process of interacting with a second circuit. This is a much more intimate linkage that may require the two circuits to be studied as a whole. In either case, the dynamic properties are not easily combined in general because the circuits are nonlinear.

Third, the separation of time scales allows some elementary circuits to be represented by transfer functions consisting of a simple power-law function. (Allometric relationships are an example of this.) This is related to the telescopic property of the S-system representation that was mentioned in Sec. III B 1. This property allows a simple block-diagram treatment of the elementary circuits that operate on a fast time scale.

\section{ACKNOWLEDGMENTS}

This work was supported in part by U.S. Public Health Service Grant No. RO1-GM30054 from the National Institutes of Health and by U.S. Department of Defense Grant No. N00014-97-1-0364 from the Office of Naval Research.

${ }^{1}$ F. Jacob and J. Monod, "Genetic regulatory mechanisms in the synthesis of proteins," J. Mol. Biol. 3, 318-356 (1961).

${ }^{2}$ J. E. Hoch and T. J. Silhavy, Two-Component Signal Transduction (American Society for Microbiology, Washington, DC, 1995).

${ }^{3}$ M. A. Savageau, "Are there rules governing patterns of gene regulation?", in Theoretical Biology_Epigenetic and Evolutionary Order, edited by B. C. Goodwin and P. T. Saunders (Edinburgh University Press, Edinburgh, 1989), pp. 42-66.

${ }^{4}$ S. A. Kauffman, The Origins of Order: Self-Organization and Selection in Evolution (Oxford University Press, New York, 1993).

${ }^{5}$ K. E. Kürten and H. Beer, "Inhomogeneous Kauffman models at the borderline between order and chaos," J. Stat. Phys. 87, 929-935 (1997).

${ }^{6}$ F. R. Blattner, G. Plunkett III, C. A. Bloch, N. T. Perna, V. Burland, M. Riley, J. Collado-Vides, J. D. Glasner, C. K. Rode, G. F. Mayhew, J. Gregor, N. W. Davis, H. A. Kirkpatrick, M. A. Goeden, D. J. Rose, B. Mau, and Y. Shao, "The complete genome sequence of Escherichia coli K-12,' Science 277, 1453-1462 (1997).

${ }^{7}$ D. Thieffry, H. Salgado, A. M. Huerta, and J. Collado-Vides, "Prediction of transcriptional regulatory sites in the complete genome sequence of Escherichia coli K-12,"' Bioinformatics 14, 391-400 (1998).

${ }^{8}$ J. R. Beckwith and E. Zipser, Eds., The Lactose Operon (Cold Spring Harbor Laboratory, Cold Spring Harbor, NY, 1970).

${ }^{9}$ C. H. Yuh, H. Bolouri, and E. H. Davidson, "Genomic cis-regulatory logic: Experimental and computational analysis of a sea urchin gene,", Science 279, 1896-1902 (1998).

${ }^{10}$ W. S. Hlavacek and M. A. Savageau, "Subunit structure of regulator proteins influences the design of gene circuitry: Analysis of perfectly coupled and completely uncoupled circuits," J. Mol. Biol. 248, 739-755 (1995).

${ }^{11}$ W. S. Hlavacek and M. A. Savageau, "Rules for coupled expression of regulator and effector genes in inducible circuits," J. Mol. Biol. 255, 121-139 (1996)

${ }^{12}$ F. C. Neidhardt and M. A. Savageau, "Regulation beyond the operon," in Escherichia coli and Salmonella: Cellular and Molecular Biology, edited by F. C. Neidhardt et al. (ASM, Washington, DC, 1996), pp. 1310-1324.

${ }^{13}$ A. Arkin, J. Ross, and H. H. McAdams, "Stochastic kinetic analysis of developmental pathway bifurcation in phage $\lambda$-infected Escherichia coli cells," Genetics 149, 1633-1648 (1998).

${ }^{14}$ M. A. Savageau, "Biochemical systems analysis II. The steady state solutions for an n-pool system using a power-law approximation," J. Theor. Biol. 25, 370-379 (1969).

${ }^{15}$ M. A. Savageau, "Power-law formalism: A canonical nonlinear approach to modeling and analysis," in World Congress of Nonlinear Analysts 92, edited by V. Lakshmikantham (de Gruyter, Berlin, 1996), Vol. 4, pp. $3323-3334$

${ }^{16}$ M. A. Savageau and E. O. Voit, "Recasting nonlinear differential equations as S-systems: A canonical nonlinear form,"' Math. Biosci. 87, 83115 (1987).

${ }^{17}$ A. Salvador, "Synergism analysis of biochemical systems. I. Conceptual framework," Math. Biosci. 163, 105-129 (2000).

${ }^{18}$ A. Salvador, "Synergism analysis of biochemical systems. II. Deviations from multiplicativity," Math. Biosci. 163, 131-158 (2000).

${ }^{19}$ E. O. Voit and M. A. Savageau, "Accuracy of alternative representations for integrated biochemical systems," Biochemistry 26, 6869-6880 (1987).

${ }^{20}$ A. Sorribas and M. A. Savageau, "Strategies for representing metabolic pathways within biochemical systems theory: Reversible pathways," Math. Biosci. 94, 239-269 (1989).

${ }^{21}$ Y. Maki, D. Tominaga, M. Okamoto, W. Watanabe, and Y. Eguchi, "Development of a system for the inference of large scale genetic networks," Pacific Symposium on Biocomputing 6, 446-458 (2000).

${ }^{22}$ M. A. Savageau, "Growth of complex systems can be related to the properties of their underlying determinants," Proc. Natl. Acad. Sci. U.S.A. 76, 5413-5417 (1979).

${ }^{23}$ B. B. Mandelbrot, The Fractal Geometry of Nature (Freeman, New York, 1983).

${ }^{24}$ M. Schroeder, Fractals, Chaos, Power Laws (Freeman, New York, 1991).

${ }^{25}$ L. Strauss, Wave Generation and Shaping (McGraw-Hill, New York, 1960).

${ }^{26}$ H. W. Bode, Network Analysis and Feedback Amplifier Design (Van Nostrand, Princeton, NJ, 1945).

${ }^{27}$ M. A. Savageau, Biochemical Systems Analysis: A Study of Function and Design in Molecular Biology (Addison-Wesley, Reading, MA, 1976).

${ }^{28}$ E. O. Voit, Canonical Nonlinear Modeling: S-System Approach to Understanding Complexity (Van Nostrand Reinhold, New York, 1991).

${ }^{29}$ N. V. Torres, E. O. Voit, and C. H. González-Alcón, “'Optimization of nonlinear biotechnological processes with linear programming. Application to citric acid production in Aspergillus niger,', Biotechnol. Bioeng. 49, 247-258 (1996).

${ }^{30}$ T.-C. Ni and M. A. Savageau, "Model assessment and refinement using strategies from biochemical systems theory: Application to metabolism in human red blood cells," J. Theor. Biol. 179, 329-368 (1996).

${ }^{31}$ D. C. Lewis, "A qualitative analysis of S-systems: Hopf bifurcations,"' in Canonical Nonlinear Modeling. S-System Approach to Understanding Complexity, edited by E. O. Voit (Van Nostrand Reinhold, New York, 1991), pp. 304-344.

${ }^{32}$ D. H. Irvine and M. A. Savageau, "Efficient solution of nonlinear ordinary differential equations expressed in S-system canonical form," SIAM (Soc. Ind. Appl. Math.) J. Numer. Anal. 27, 704-735 (1990).

${ }^{33}$ S. A. Burns and A. Locascio, "A monomial-based method for solving systems of non-linear algebraic equations," Int. J. Numer. Methods Eng. 31, 1295-1318 (1991).

${ }^{34}$ M. A. Savageau, "Finding multiple roots of nonlinear algebraic equations using S-system methodology," Appl. Math. Comput. 55, 187-199 (1993).

${ }^{35}$ M. A. Savageau, "The behavior of intact biochemical control systems," Curr. Top. Cell. Regul. 6, 63-130 (1972).

${ }^{36} \mathrm{M}$. A. Savageau, "A theory of alternative designs for biochemical control systems," Biomed. Biochim. Acta 44, 875-880 (1985).

${ }^{37}$ R. Alves and M. A. Savageau, "Extending the method of mathematically controlled comparison to include numerical comparisons," Bioinformatics 16, 786-798 (2000).

${ }^{38}$ M. A. Savageau, "Design of molecular control mechanisms and the demand for gene expression," Proc. Natl. Acad. Sci. U.S.A. 74, 5647-5651 (1977).

${ }^{39}$ M. A. Savageau, "Models of gene function: general methods of kinetic analysis and specific ecological correlates," in Foundations of Biochemical Engineering: Kinetics and Thermodynamics in Biological Systems, edited by H. W. Blanch, E. T. Papoutsakis, and G. N. Stephanopoulos (American Chemical Society, Washington, DC, 1983), pp. 3-25.

${ }^{40}$ M. A. Savageau, "Regulation of differentiated cell-specific functions," Proc. Natl. Acad. Sci. U.S.A. 80, 1411-1415 (1983).

${ }^{41}$ V. I. Arnol'd, Catastrophe Theory (Springer-Verlag, New York, 1992).

${ }^{42}$ A. Novick and M. Weiner, "Enzyme induction as an all-or-none phenomenon,” Proc. Natl. Acad. Sci. U.S.A. 43, 553-566 (1957).

${ }^{43}$ J. R. Sadler and A. Novick, "The properties of repressor and the kinetics of its action,"' J. Mol. Biol. 12, 305-327 (1965). 
${ }^{44}$ E. M. Cooke, Escherichia coli and Man (Churchill Livingstone, London, 1974).

${ }^{45}$ M. A. Savageau, "Demand theory of gene regulation: Quantitative development of the theory,"' Genetics 149, 1665-1676 (1998).

${ }^{46}$ M. A. Savageau, "Demand theory of gene regulation: Quantitative application to the lactose and maltose operons of Escherichia coli," Genetics 149, 1677-1691 (1998).

${ }^{47}$ J. H. Bond and M. D. Levitt, "Quantitative measurement of lactose absorption," Gastroenterology 70, 1058-1062 (1976).

${ }^{48}$ J.-R. Malagelada, J. S. Robertson, M. L. Brown, M. Remington, J. A. Duenes, G. M. Thomforde, and P. W. Carryer, "Intestinal transit of solid and liquid components of a meal in health,' Gastroenterology 87, 12551263 (1984).

${ }^{49}$ J. H. Cummings and H. S. Wiggins, "Transit through the gut measured by analysis of a single stool," Gut 17, 219-223 (1976).

${ }^{50}$ J. S. S. Gear, A. J. M. Brodribb, A. Ware, and J. T. Mann, "Fiber and bowel transit times," Br. J. Nutr. 45, 77-82 (1980).

${ }^{51}$ M. A. Savageau, "Escherichia coli habitats, cell types, and molecular mechanisms of gene control," Am. Nat. 122, 732-744 (1983).

${ }^{52}$ L. Hayflick, "The cellular basis for biological aging," in Handbook of the Biology of Aging, edited by C. E. Finch and L. Hayflick (Van Nostrand Reinhold, New York, 1977), pp. 159-186.

${ }^{53}$ H. I. Sears, I. Brownlee, and J. K. Uchiyama, "Persistence of individual strains of $E$. coli in the intestinal tract of man," J. Bacteriol. 59, 293-301 (1950).

${ }^{54}$ H. I. Sears and I. Brownlee, "Further observations on the persistence of individual strains of Escherichia coli in the intestinal tract of man," J. Bacteriol. 63, 47-57 (1952).

${ }^{55}$ D. A. Caugant, B. R. Levin, and R. K. Selander, "Genetic diversity and temporal variation in the E. coli population of a human host,' Genetics 98, 467-490 (1981).

${ }^{56} \mathrm{~S}$. Garges, "Activation of transcription in Escherichia coli: The cyclic AMP receptor protein," in Transcription: Mechanisms and Regulation, edited by R. C. Conaway and J. W. Conaway (Raven, New York, 1994), pp. 343-352. 